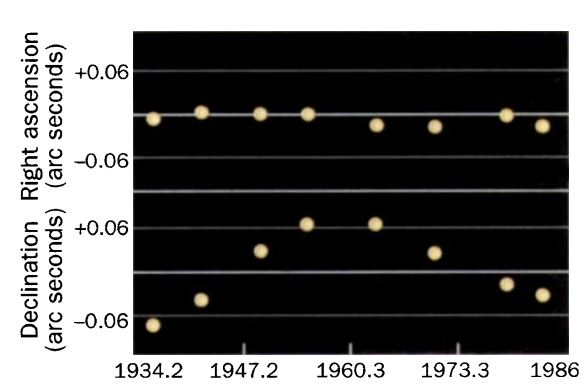

FIG. 2 The motion of Lalande 21185 from 50 years of photographic data taken at the Allegheny Observatory, after correction for the effects of parallax and proper motion. The points are 6-year means. Although most of the curvature is caused by perspective acceleration, it departs significantly from that predicted. (Figure kindly supplied by G. Gatewood.)

it would have been seen long ago.

The innermost companion, for which the orbit appears to be circular, would have a maximum separation of about 1 arc second from the primary. That makes direct imaging a real possibility, despite the glare from the star, using a large ground-based telescope (with a mirror greater than 3 metres in diameter) at a good site, or the Hubble Space Telescope. Only by capturing an image of such an object and eventually securing a spectrogram can we be sure it is a planet.

If it shines largely by reflected light, the image of the 5.8-year planet may only be some $10^{-9}$ as bright as the star. But with the introduction of adaptive optics and the refurbishment of the Hubble Space Telescope, the quality of images that can now be achieved both from the ground and from space at near-infrared wavelengths ( 1 to $2 \mu \mathrm{m}$ ) has recently improved enough to make detection feasible.

Diffraction sets the ultimate limit to angular resolution, but, on the ground, image-spread is dominated by rapidly changing thermal turbulence in the atmosphere. Thanks to adaptive optics, however, wavefront distortions can be sensed and corrected in real time with a deformable mirror. This, in principle, makes neardiffraction-limited imaging possible, at least at near-infrared wavelengths. Several such adaptive optical systems of differing complexity are now in use on large ground-based telescopes, and some have coronagraphic optics which exclude the great majority of contaminating light from the star. So the race is on to obtain a convincing image of a planet in the Lalande 21185 system, a race that might be won as early as next year.

Gordon A. H. Walker is in the Physics and Astronomy Department, University of British Columbia, Vancouver, British Columbia, Canada V6T $1 Z 4$.

\footnotetext{
1. Mayor, M. \& Queloz, D. Nature 378, 355-359 (1995).

2. Marcy, G. W. \& Butler, R. P. http://cannon.sfsu.edu/

$\sim$ williams/planetsearch/planetsearch.html

3. Gatewood, G. Astr. J. 94, 213-224 (1987).
}

\title{
Changing times, changing teams
}

\section{Henry Gee}

LAST Wednesday (26 June), England was beaten by Germany in the semi-finals of the European soccer championships, Euro '96. The match was settled by a penalty shoot-out, and was an uncanny replay of the 1990 World Cup - a semifinal win for Germany over England, again decided by penalties. But the parallels run deeper still: through a World Cup quarterfinal meeting in 1970 (Germany the winners), and back to the 1966 World Cup final, with England this time the victor.

But how real are the comparisons? Nominally, the teams represented the same adversaries, but the individual players were different. Of those on the pitch last Wednesday, only five played in 1990, and, of course, there were none from 1970 and 1966. Indeed, the England captain in 1966, Bobby Moore, died recently, and most of the current squads weren't even born then. This is to be expected, as national teams carry on, eclipsing individual careers.

If soccer were as we might assume ecology to be, Bobby Moore would have led the England team last Wednesday: it is easy to think that the faunal associations we see today are as they have ever been, the individual members bound together in equilibrium by links of adaptation and competition. Communities, one assumes, respond to factors such as climate change by wholesale shifts. For example, cooling would drive deciduous forests into southern refugia, and their animal residents, the whole team of them, with them.

Palaeontologists have long known that history, like soccer, tells a different story, but this has probably never been documented in the exhaustive detail that Graham et al. have achieved in their study of the faunal history of the United States over the past 20,000 years (Science 272, 1601-1606; 1996). They have collated species lists of mammal faunas from 2,945 localities in the contiguous United States, and have developed a software package called FAUNMAP which displays this information in space and time.

Individual species can be picked out, and their ranges followed as the climate changes. Associations between species can also be measured, using modern examples as standards. This has allowed researchers to find out whether modern-day ecological associations have long and distinct histories, or have been thrown together more recently. FAUNMAP can also be used to measure spatial heterogeneity; that is, the extent to which faunal associations differ with distance.

FAUNMAP analyses show that species are not team players - they tend to look after themselves, not their associations, and respond to climate change in idiosyn- cratic ways. At the end of the Pleistocene, $10,000-15,000$ years ago, some species moved northwards, but at different rates. Others, though, moved eastwards, while the ranges of some western species contracted. Still others have stayed put, their present distribution being the same as it was during the coldest parts of the ice age 15,000-20,000 years ago.

The implications of this work are twofold. First, the uncoordinated movement of species in response to climate change produces novel combinations, makeshift alliances rather than associations forged by co-evolution and competition over millions of years. Second, the ecological stability we see today is illusory modern communities emerged only in the past few thousand years.

Answers to larger questions of biogeography and spatial heterogeneity are sensitive to scale. At the largest scales, Graham et al. show that present-day biogeographical 'provinces' have deep roots, even though many of the participants have changed. The faunas of the United States appear to be divided into eastern and western components along the 100-degree line of longitude, whether one looks at Late Pleistocene or Holocene faunas (respectively before and since the conventional 10,000-year watershed). This division marks a boundary between the forests and grasslands to the east, and the deserts and mountains to the west.

Closer inspection reveals significant differences. For example, the Late Pleistocene faunas of the eastern United States are divided into distinct northern and southern components, presumably dictated by temperature. Even so, within any given province, one can use the roster of species to discern environmental preferences, even if the membership of each list differs from place to place. There'll always be English and German soccer teams (at least, one hopes so). But the individual players will have changed.

Faunas from any two places share progressively fewer species with increasing distance between the localities, whether one is looking at Late Pleistocene or Holocene faunas. But for any given distance, Late Pleistocene faunas are less similar than Holocene faunas. This suggests that spatial heterogeneity in the Pleistocene was greater than today. Faunas responded to a more intricate patchwork of environments with a greater variety of species. The environment of the modern United States is more homogeneous, and there are fewer distinct faunal associations - in effect, fewer teams are up for the cup.

Henry Gee is an assistant editor at Nature. 